\title{
FCC-ee: the synthesis of a long history of e+e- circular colliders
}

\author{
K. Oide ${ }^{1}$, J. Wenninger ${ }^{2, a}$ iD \\ ${ }^{1}$ KEK, Tsukuba, Ibaraki, Japan \\ 2 CERN, Geneva, Switzerland
}

Received: 8 June 2021 / Accepted: 26 August 2021

(C) The Author(s) 2021, corrected publication 2022

\begin{abstract}
The design of FCC-ee is relying on the accumulated experience of $\mathrm{e}^{+} \mathrm{e}^{-}$colliders that have been designed, constructed and operated in the past 40 years. FCC-ee will surpass the $26.7 \mathrm{~km}$ long Large Electron Positron collider LEP by a factor 4 in size. Like for LEP the large size is justified by the need to control the synchrotron radiation losses that for both machines reach a few percent per turn. To that end LEP had the first large superconducting (SC) RF system with around $3.8 \mathrm{GV}$ of accelerating voltage. LEP achieved for the first time very large beam-beam parameters of around 0.08 , and it relied on transversely polarized beams to determine accurately the beam energy for the experiments. The DA $\Phi$ NE collider, together with PEP II and KEKB split the two beams into separate vacuum chambers to reach much higher Ampere-level beam currents. To overcome beam-beam lifetime and performance issues DA $\Phi$ NE used for the first time the Crab Waist concept for the interaction region (IR) optics. The B-factories, PEP-II and KEKB have verified the double-ring $\mathrm{e}^{+} \mathrm{e}^{-}$ collider with multi-ampere stored currents for over 1000 bunches, small $\beta^{*}$, top-up injection, and achieved then-highest luminosity. KEKB has applied 22-mrad crossing angle at the IP with crab crossing. Both machines inherited accelerator techniques from their predecessors, PEP and TRISTAN, which was a small-scale LEP. Currently the next generation SuperKEKB collider is starting up. It has already achieved some milestones required for FCC-ee such as small $\beta^{*}(0.8 \mathrm{~mm})$ and virtual crab-waist scheme with a large Piwinski angle $(>10)$.
\end{abstract}

\section{Introduction}

The FCC-ee collider [1] is designed to operate over the beam energy range of $40 \mathrm{GeV}$ to $185 \mathrm{GeV}$ as $\mathrm{Z}, \mathrm{W}$, Higgs and top factory. The collider will operate at constant synchrotron radiation power, and due to the steep dependence of energy loss on energy, the resulting beam currents range from Ampere-level with tens of thousand of bunches around $45 \mathrm{GeV}$ to a few milliamperes with a few bunches at $185 \mathrm{GeV}$.

The FCC-ee design [2] relies on the accumulated experience of $\mathrm{e}^{+} \mathrm{e}^{-}$colliders that have been designed, constructed and operated in the past 40 years. FCC-ee will surpass the $26.7 \mathrm{~km}$ long Large Electron Positron collider LEP [3] by a factor 4 in size, justified by the need to control the synchrotron radiation losses reaching a few percent per turn for operation at

\footnotetext{
a e-mail: jorg.wenninger@ cern.ch (corresponding author) URL: https://www.kek.jp/en/
} 
the $\bar{t} t$ threshold. The two beams will circulate in separate vacuum chambers following the examples of other modern high luminosity machines PEP II [4], KEKB [5], superKEKB $[6]$ and DA $\Phi N E$ [7]. This will allow FCC-ee to reach Ampere-level beam currents without long range beam-beam limitation as was the case for LEP. The Crab Waist concept for the interaction region (IR), introduced at $\mathrm{DA} \Phi \mathrm{NE}$, will help overcome beam-beam limitations.

\section{LEP: 1989-2000}

The Large Electron Position collider LEP is the $\mathrm{e}^{+} \mathrm{e}^{-}$collider with the largest size and with the highest beam energy of just over $104 \mathrm{GeV}$. Four large experiments, L3, ALEPH, OPAL and DELPHI were installed around the ring with a fourfold symmetry. The LEP magnet system was designed for a maximum beam energy of around $130 \mathrm{GeV}$ which would have been sufficient to reach the $\mathrm{ZH}$ production threshold. LEP was designed as a single ring collider with four bunches per beam. It operated with relatively large IP betatron functions of $\beta_{\mathrm{x}}=1.25-2.5 \mathrm{~m}$ and $\beta_{\mathrm{y}}=4-10 \mathrm{~cm}$ compared to latest generation B-factory colliders.

\subsection{LEP1: the $Z$ boson years}

LEP operation began in July 1989 at the $Z$ pole which corresponds to a beam energy close to $45 \mathrm{GeV}$. LEP operated at and around the $Z$ pole until 1995 to study the $Z$ boson including its resonance line-shape.

The initial operation with 4 bunches per beam was upgraded to 8 bunches per beam with a pretzel orbit scheme spanning all 8 LEP arcs in 1993. In 1995, the number of bunches was increased to 12 per beam using short bunch trains with local separation schemes around the four IPs. The vertical beam-beam tune shift was limited to around 0.04 , and most of the time LEP operated at the beam-beam limit using emittance controlling wigglers to maintain a near constant beam-beam tune shift as a function of the bunch current. Controlling the backgrounds in the experiments was a delicate task. The peak luminosity reached $\mathcal{L}=2.4 \times 10^{31} \mathrm{~cm}^{-2} \mathrm{~s}^{-1}$ in bunch train operation in 1995 [3].

The precise determination of the $Z$ boson mass and width depended on an accurate determination of the centre-of-mass energy at each IP [8]. The fundamental ingredient was the measurement of the beam energy using resonant depolarization, with a typical relative accuracy of $2 \times 10^{-5}$ for an individual measurement. Such measurements were however not compatible with luminosity operation, because transverse polarization required different working points and machine setups, they had to be performed at the end of luminosity fills or in dedicated sessions. Two major measurement campaigns were performed in 1993 and 1995 which unveiled many effects impacting the beam energy, the most prominent effects were earth tides, long-term geological deformations of the ring, earth current from DC-railway trains. Reconstruction of the centre-of-mass energy required further corrections due to the RF system voltage distribution, phase and alignment errors. Finally, the beam offsets at the IP had to be controlled with high accuracy to avoid biases due to dispersion of opposite sign for the two beams at the IP. The final contribution of the beam energy to the uncertainty on the $\mathrm{Z}$ boson mass of $91.18 \mathrm{GeV}$ is $\pm 1.9 \mathrm{MeV}$ [8].

\subsection{LEP2: the $W$ and Higgs boson years}

Operation of LEP2 spanned the years 1996 to 2000. While the first years were dedicated to studying the $\mathrm{W}$ boson at ever increasing beam energies, in particular the precise measurement 


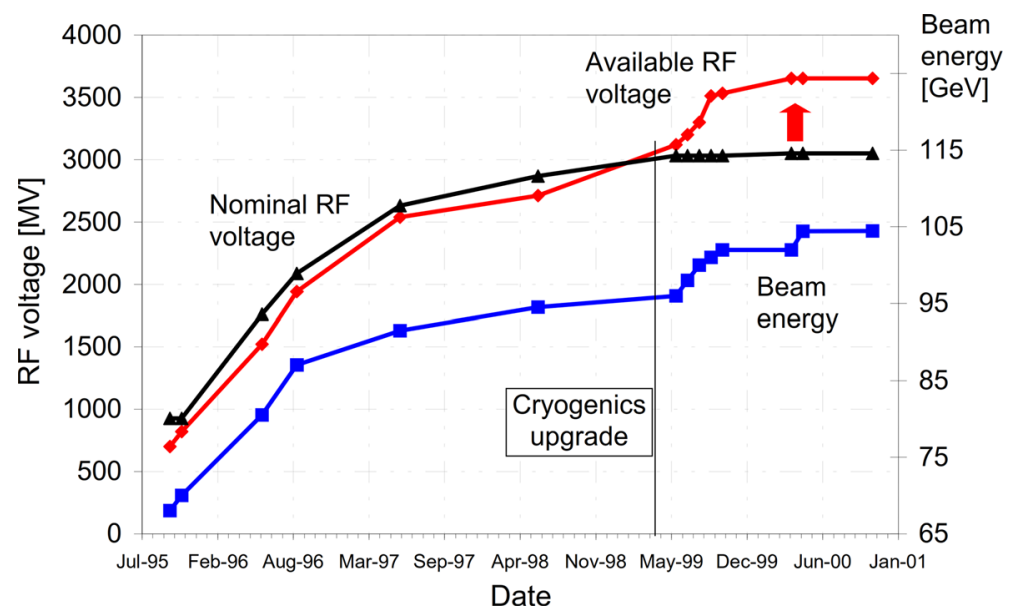

Fig. 1 Evolution of the nominal and the available LEP RF voltage as well as the beam energy over time between 1996 and 2002. Starting in 1999 the RF cavity gradient was pushed beyond its design value providing more RF voltage than the nominal design voltage

of the $\mathrm{W}$ mass as a continuation of the $Z$ boson measurements [9], the focus shifted to the hunt for the Higgs boson in the last 2 years, with the aim of pushing the energy reach to the maximum. In the last year, some peak luminosity was traded for a slight increase in energy reach.

The first superconducting RF modules were installed in 1994 while LEP was operating at the $Z$ pole. In the following years the number of modules steadily increased up to $72 \mathrm{RF}$ modules. In the final configuration of LEP in 2000, $288 \mathrm{Nb}$-Ti thin film cavities with an active length of $490 \mathrm{~m}$ and 56 room-temperature Copper cavities, powered by 43 klystrons of 1 to $1.3 \mathrm{MW}$, provided a peak RF voltage of $3.6 \mathrm{GV}$ as shown in Fig. 1 [10]. If LEP had been equipped completely with SC cavities using all available space in the four RF straight sections, the peak RF voltage could have reached $4.8 \mathrm{GV}$ pushing the energy reach to $110 \mathrm{GeV}$ per beam [11]. To be able to reach the HZ production threshold the beam energy should have been pushed to $114 \mathrm{GeV}$, in reach of the magnet system but not of the most optimistic RF configuration.

Until 1999 the energy reach of LEP was extended by adding cavities operated at around $6 \mathrm{MV} / \mathrm{m}$, then the RF voltage was pushed by operating the cavities at an average gradient of up to $7.5 \mathrm{MV} / \mathrm{m}$. The maximum usable energy in 2000 was between 104 and $104.5 \mathrm{GeV}$ per beam.

The peak performance of LEP2 was achieved in 1998 and 1999 with a beam current of $6.2 \mathrm{~mA}$ and 4 bunches per beam. The peak luminosity reached $1.07 \times 10^{32} \mathrm{~cm}^{-2} \mathrm{~s}^{-1}$ as shown in Figure 2. The vertical beam-beam tune shift reached 0.083 without signs of significant saturation, extrapolations hinted at a vertical beam-beam tune shift limit around 0.12 , a limit that was never reached since the RF system could not provide sufficient power for higher beam currents. The performance was optimized using a low emittance lattice with a horizontal phase advance of $102^{\circ}$ and a vertical phase advance of $90^{\circ}$ which provided the best dynamic aperture [3]. Furthermore, the horizontal damping partition number was increased up to a value of 1.8 at $94 \mathrm{GeV}$ to lower the horizontal emittance and boost the performance. This was achieved by operating the machine with a RF frequency shift relative to the nominal frequency for which the beam would have been centered radially. 

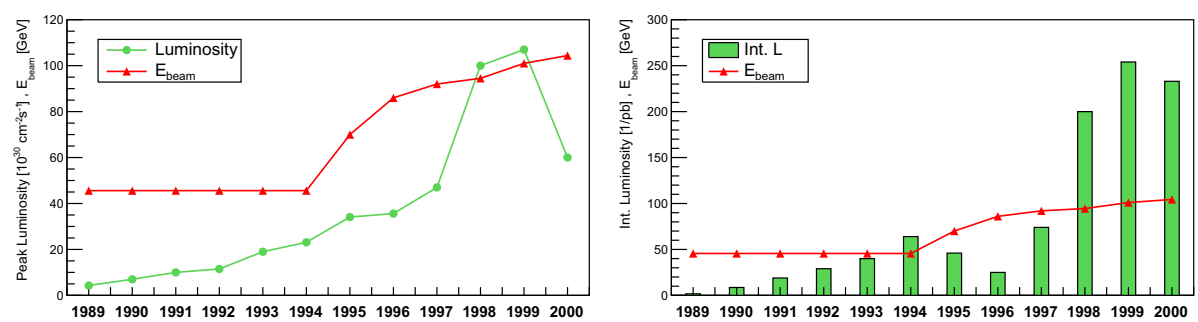

Fig. 2 Evolution of the LEP peak luminosity (left), integrated luminosity (right) and energy over time. In the last year of operation, peak luminosity was traded for the maximum reach in energy

\section{The $\tau$ and B factories: from the mid 1990's until today}

A number of machines belong to this category of high stored current and high luminosity colliders: CESR, DA $\Phi$ NE, PEP-II, KEKB, BEPC-II, VEPP2000 and SuperKEKB. New features, both at the technical and the theoretical level, have been observed, investigated, and verified through the success of these machines which employ several new schemes.

\subsection{Machine design}

Double ring colliders became the new norm to store high beam currents and a large number of bunches (DA $\Phi$ NE, PEP-II, KEKB, BEPC-II, SuperKEKB), avoiding limitations due to long range beam-beam in the machine sections outside the interaction regions, inheriting the histories of DORIS and ISR.

Top-up injection is used to maintain nearly constant beam currents over time (PEP-II, $\mathrm{KEKB}, \mathrm{DA} \Phi \mathrm{NE}, \mathrm{BEPC}-\mathrm{II}$, SuperKEKB). As a consequence the machines become very stable since the synchrotron radiation (SR) and the associated component heating remain constant. An example is shown in Fig. 3 for KEKB. Top-up schemes were made possible by fully utilizing the injector capacities including the positron source.

Collisions with crossing angles became a standard of modern colliders (DA $\Phi$ NE, KEKB, BEPC-II, SuperKEKB). The crab crossing (KEKB) and crab waist schemes (DA $\Phi$ NE [12], SuperKEKB) are two derived concepts. The former will be adapted to upcoming hadron machines such as HL-LHC and EIC. The latter has been successfully used and contributes to the performance improvements.

The vertical $\beta^{*}$ was squeezed down to $\beta_{y}^{*}=6 \mathrm{~mm}$ at KEKB and $0.8 \mathrm{~mm}$ at SuperKEKB, which was only possible with an accurate modeling and correction of the beam optics including detector solenoids oriented at a large tilt angle with respect to the beam directions.

High beam-beam parameters of $\sim 0.1$ have been obtained at PEP-II and KEKB, similar to LEP2 despite much lower radiation damping, thanks to precise control of the machine and the beam optics. A horizontal tune very close to the half-integer resonance $\left(v_{x} \sim 0.506\right)$ could be used at KEKB, PEP-II, and CESR to fully profit from the dynamic- $\beta$ effect due to the strong head-on beam-beam force. Feedback systems to maintain the collision have been developed based on beam-beam kicks and luminosity dithering at PEP-II.

\subsection{High beam currents}

These machines had to overcome issues due to the high stored beam currents of $1-3 \mathrm{~A}$ with close to or over thousand bunches (DA $\Phi$ NE [13], PEP-II [4], KEKB [5]). Although some 


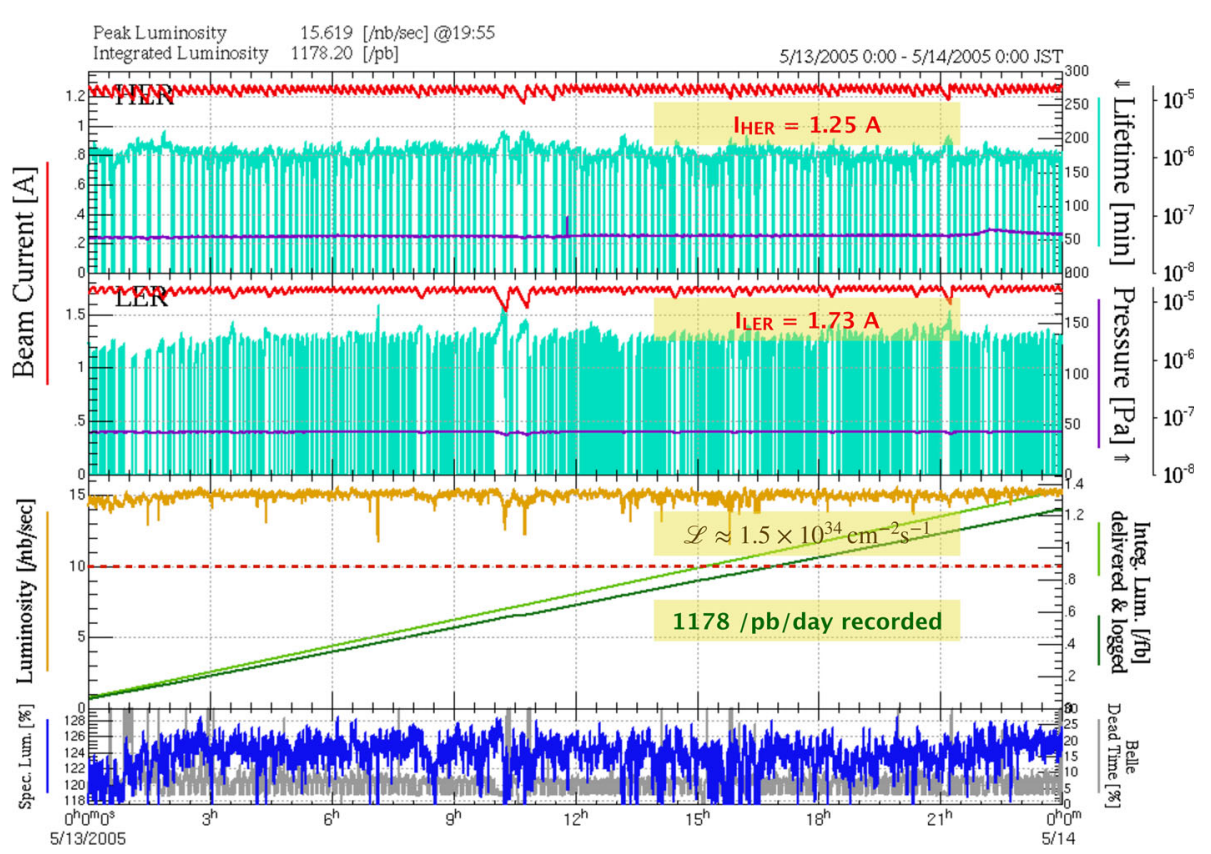

Fig. 3 An example of KEKB with top-up injection over one day in 2005. The two top plots represent the beam currents (red), beam lifetimes (green) and the vacuum pressures (blue) of the $e^{-}$and $e^{+}$rings. The two lower plots represent the instantaneous (orange) and the integrated (green) luminosity, the specific luminosity (blue, luminosity per bunch divided by the product of bunch currents) as well as the Belle detector dead-time (grey)

hadron machines stored similar or even higher currents, electron machines have difficulties due to synchrotron radiation and very short bunch length compared to hadron beams.

Vacuum components had to comply with the high stored current, for example shielded bellows, pumping ports, gate valves, buttons for beam position monitor. Many incidents with component overheating due to high synchrotron radiation power and HOMs were experienced on these components.

Electron clouds developed in the positron rings of the KEKB and PEP-II B-factories. This was the most serious issue at KEKB as it led to large blowup of vertical beam size. The blowup was caused by a single-bunch TMCI-like instability, which was driven by the electron cloud generated by the high stored positive charge [14]. Many cures such as additional solenoid fields wounds around the vacuum chambers over the entire ring, TiN coating, antechambers, electrostatic fields, grooved surfaces, etc., have been successfully applied to fight electron clouds. As the result, electron clouds are no longer an issue at SuperKEKB [15] up to a stored beam current of $1 \mathrm{~A}$.

To suppress beam instabilities in the presence of high stored current and many bunches, the RF systems had to be redesigned to store the high currents. The reaction to the beam loading on the fundamental mode of the RF cavity was the first thing to overcome. This was achieved with single-cell superconducting cavities (CESR, KEKB, BEPC-II), copper cavities with energy storage (KEKB), or damped cavities with active RF feedback (PEPII). To suppress coupled bunch instabilities, bunch-by-bunch feedback systems have been deployed. Transverse feedback systems were employed in all machines, while a longitudinal feedback system was also necessary at PEP-II, BEPCII and DA $\Phi$ NE. 


\subsection{Machine-detector interfaces}

Detector and accelerator are strongly coupled at the interaction point of those machines, they cannot be designed independently based on a predefined boundary. Accelerator and detector physicists must work hand-in-hand to understand the most critical issues on the both sides. Once designed, the interaction region is the most difficult part of the project to modify at a later stage. All issues must be considered and taken into account from the beginning of the project.

Beam backgrounds in the detectors were among the most difficult figures to predict in advance. A large amount of redundancy, e.g. in the collimation system, was necessary to cope with the observed backgrounds. With top-up injection the injector can also become a source of beam background to the detector. An important lesson is that background optimization and beam collimation must not be designed independently of the other ring components [16]. The collimators must not only protect the detector, but also accelerator components such as final focus superconducting magnets. As a side effect collimators generate additional impedances that can limit operation at high beam current.

The experience of LEP in terms of beam backgrounds, namely that the energy of the photons emitted by dipoles shining directly on the vacuum chambers in the straight section should not exceed $100 \mathrm{keV}$, was fed directly into the FCC-ee geometry in the form of an asymmetric straight section layout with soft bending on the incoming beam path and stronger bending on the outgoing path.

\section{Conclusion}

Layout and optics of the FCC-ee collider [1,2] take advantage of advances in accelerator design and operation that took place over the last 430 years. FCC-ee will push the size of the collider by a factor four with respect to LEP, based on the experience of operating a huge collider and a large RF system, as well as exquisite accuracy on the beam energy measurements. A modern two-ring design was adopted from the beginning to reach Amperelevel currents without limitations due to long range beam-beam which is particularly well suited for the $Z$ and $W$-factory running scenarios. Advances on the control of the beam-beam effects using the Crab waist scheme and well as the interaction region design experiences of KEKB, SuperKEKB and LEP integrated into the interaction region layout and optics.

Funding Open Access funding provided by CERN. This project is co-funded from the European Union's Horizon 2020 research and innovation programme under grant agreement No 951754.

Data Availability Statement No datasets were generated or analysed for this article. Raw data for the LEP Figs. 1 and 2 is available from the authors.

Open Access This article is licensed under a Creative Commons Attribution 4.0 International License, which permits use, sharing, adaptation, distribution and reproduction in any medium or format, as long as you give appropriate credit to the original author(s) and the source, provide a link to the Creative Commons licence, and indicate if changes were made. The images or other third party material in this article are included in the article's Creative Commons licence, unless indicated otherwise in a credit line to the material. If material is not included in the article's Creative Commons licence and your intended use is not permitted by statutory regulation or exceeds the permitted use, you will need to obtain permission directly from the copyright holder. To view a copy of this licence, visit http://creativecommons.org/licenses/by/4.0/. 


\section{References}

1. A. Abada et al., (Future Circular Collider Study), FCC-ee: The Lepton Collider. Eur. Phys. J. Special Topics 228, 261-623 (2019)

2. K. Oide et al., Design of beam optics for the future circular e+e- collider rings. Phys. Rev. Accel. Beams 19, 111005 (2016)

3. D. Brandt et al., Accelerator Physics at LEP. Rep. Prog. Phys. 63, 939-1000 (2000)

4. J. Seeman, Last year of PEP-II B-factory operation, Proceedings, 11 th european conference, EPAC 2008, Genoa, Italy, June 23-27 (2008)

5. K. Oide, K.E.K.B. B-factory, the luminosity frontier. Prog. Theor. Phys. 122, 69-80 (2009)

6. K. Akai, K. Furukawa, H. Koiso, SuperKEKB collider. Nuclear Instrum. Methods Phys. Res. Sect. A 907, 188-199 (2018)

7. C. Milardi et al, DAFNE setup and operation with the crab-waist collision scheme, Proceedings, 11th European Conference, EPAC 2008, Genoa, Italy, June 23-27, (2008)

8. R. Assmann et al., Calibration of centre-of-mass energies at LEP1 for precise measurements of Z properties. Eur. Phys. J. C 6(2), 187-223 (1999)

9. R. Assmann et al., Calibration of centre-of-mass energies at LEP2 for a precise measurement of the $\mathrm{W}$ boson mass. Eur. Phys. J. C 39, 253-292 (2005)

10. R. Assmann, M. Lamont, S. Myers, A brief history of the LEP collider. Nucl. Phys. B. , Proc. Suppl. 109, 17 (2002)

11. K. Hubner, Designing and Building LEP, CERN-AB-2004-099 ADM (2004)

12. M. Zobov et al., Test of "Crab-Waist" Collisions at the DA $\Phi$ NE. Phys. Rev. Lett. 104, 174801 (2010)

13. C. Milardi, D. Alesini, A. Drago, A. Gallo, A. Ghigo, S. Guiducci, P. Raimondi, M. Serio, A. Stella, M. Zobov. https://doi.org/10.18429/JACoW-eeFACT2018-TUYAA01

14. K. Ohmi, F. Zimmermann, Head - tail instability caused by electron cloud in positron storage rings. Phys. Rev. Lett. 85, 3821-3824 (2000)

15. Y. Suetsugu, H. Fukuma, K. Ohmi, M. Tobiyama, K. Shibata, CERN Yellow Rep. Conf. Proc. 7, 201-208 (2020). https://doi.org/10.23732/CYRCP-2020-007.201

16. H. Tanigawa et al., Belle-II SVD. Nucl. Instrum. Meth. A 982, 164580 (2020). https://doi.org/10.1016/j. nima.2020.164580 